\title{
Assessment of Diabetic Patient Perception on Diabetic Disease and Self-Care Practice in Dilla University Referral Hospital, South Ethiopia
}

Yohannes Addisu ${ }^{1}$, Akine Eshete ${ }^{1}$ and Endalew Hailu ${ }^{2 *}$

${ }^{1}$ College of Health and Medical Sciences, Dilla University, Ethiopia

${ }^{2}$ Department of Nursing, College of Public Health and Medical Sciences, Jimma University, Ethiopia

\begin{abstract}
Background: Diabetes is a chronic and irreversible disease that lasts, lifelong, directly concerns any individual of all ages and their relatives, and brings heavy economic burden, affects self-care activities and shortens life expectancy due to the chronic damages it causes, Thus, before considering any possible intervention it was imperative to assess present knowledge, perception, and self-care practices of patients towards the management of diabetes.

Methods: A facility based cross-sectional study supplemented by using both quantitative and qualitative methods was conducted from April - June 2013 in Dilla referral hospital. A total of 310 participants with Diabetes Mellitus were interviewed. Face-to-face interviews were used for quantitative data; and qualitative data were collected by in-depth interview. SPSS version 20 was used to perform descriptive and logistic regression analyses. Statistical significance was set at $P$-value $<0.05$ to judge the association.

Result: Two third, 238 (76.8) of them had good practiced on the recommended self-care practices. Among the recommended self-care behaviors, drug adherence $289(93.2 \%)$, dietary intake $154(49.7 \%)$ and regular exercise $138(44.5 \%)$ were the most practiced self-care. Self-blood glucose monitoring was the least practiced which accounted $62(20 \%)$. Approximately $78 \%$ of diabetic patients were developed positive perception towards $\mathrm{DM}$ and has a significant effect for patients with diabetes to provide own self-care practice [OR-2.74, $95 \% \mathrm{Cl}(1.27$, 5.91)]. Majority $79.4 \%$ of the respondents were knowledgeable about diabetes, but those diabetic patients who were knowledgeable on DM were less likely performed recommended self-care to manage the disease [OR-0.29, $95 \% \mathrm{Cl}(0.10,0.80)]$. On other hand those diabetic patients who were knowledgeable on DM self-care were more likely performed recommended self-care [OR-6.52, $95 \% \mathrm{Cl}(2.88,14.78)]$. Education also has a significant effect for patients with diabetes in their own self-care practice. A major point to address therefore is regular access to/contact with diabetic educators which currently is severely substandard.

Conclusions: Management of diabetes may be enhanced by reinforcing patients' knowledge, developing positive perception and encouraging behavior change whilst taking into consideration patients' backgrounds. To increase the self-care behavior, the health care team should be utilizing a patient-centered approach in order to deliver diabetes messages on specific issues of management practice.
\end{abstract}

Keywords: Diabetes; Insulin; Blood glucose; Alcohol; Smoking

\section{Introduction}

\section{Background}

Diabetesmellitus (DM) is "agroup of metabolicdiseasescharacterized by hyperglycemia resulting from defects in insulin secretion, insulin action or both" (American diabetes association, 2005), manifested by carbohydrates, fat, protein metabolism abnormality. It is a chronic disease, which has no cure and associated with high rate of morbidity and mortality in both developing and developed countries and also becoming a pandemic in the world, with increased need for health care $[1,2] . \mathrm{DM}$ is increasingly prevalent and one of the top public health concerns all over the world. The International Diabetes Federation (IDF) estimates that 23 million years of life are lost due to disability and reduced quality of life as a result of complications associated with diabetes. Evidence has shown that $\$ 232$ billion U.S. dollars were spent worldwide in 2007 to treat and prevent diabetes. This figure is expected to climb to a minimum of over $\$ 300$ billion in 2025 [3]. The Diabetic Prevention Programs (DPP) found conclusively that with moderate exercise and change in diet people can reduce the risk of developing type 2 diabetes by $58 \%$. Sub-Saharan Africa, like the rest of the world, is experiencing an increasing prevalence of diabetes alongside other noncommunicable diseases [2].

Even though the actual number was not known, World health organization has estimated the number of diabetic cases in Ethiopia to be 800,000 by the year 2000 , and the number is expected to increase to 1.8 million by 2030 [4]. To prevent serious morbidity and mortality, diabetes treatment requires dedication to demanding self-care behaviors in multiple domains, including food choices, physical activity, proper medications intake and blood glucose monitoring [5]. Self-care in diabetes is a process where the person attempts a variety of self-care strategies, according to her/his unique body's cues, until discovering what is effective for her/his lifestyle and contextual situation and is individuals' taking the necessary action to protect their lives, health and well-being [6-8]. In order to control their disease, patients with diabetes need to adopt self-care activities such as exercising, an appropriate diet, regular exercises, control of blood glucose, appropriate use of oral antidiabetics, awareness of the effects and side effects of insulin treatment, avoiding alcohol use and smoking, preventing complications of diabetes, and compliance to life-long medication $[9,10]$.

In addition there are no studies that have addressed the assessment

*Corresponding author: Endalew Hailu, Department of Nursing, College of Public Health and Medical Sciences, Jimma University, Ethiopia, Tel: 251911048847; E-mail: endale.10@gmail.com

Received September 04, 2014; Accepted September 27, 2014; Published October 01, 2014

Citation: Addisu Y, Eshete A, Hailu E (2014) Assessment of Diabetic Patient Perception on Diabetic Disease and Self-Care Practice in Dilla University Referral Hospital, South Ethiopia. J Metabolic Synd 3: 166. doi:10.4172/2167-0943.1000166

Copyright: ( 2014 Addisu Y, et al. This is an open-access article distributed under the terms of the Creative Commons Attribution License, which permits unrestricted use, distribution, and reproduction in any medium, provided the original author and source are credited. 
of diabetic patient perception on diabetic disease and self-care practice in Dilla university referral hospital, South Ethiopia

Hence this study is designed to address this gap. So, the purpose of this study is assess the perception of diabetic patients about diabetic related education and self-care practice in in Dilla university, referral hospital, Southwest Ethiopia.

\section{Significance of the Study}

Diabetes is a chronic disease that requires lifelong self-care behavior, today, successful treatment of diabetes mellitus is closely associated with the education of both Patients and their relatives and patient education is one of the most important responsibilities of health professions. Educators emphasize that learning depends on the individual's level of development, and patients should have awareness in diabetes education. In the management of diabetes, helping patients improve their health and quality of life is considered an important aspect of diabetes self-care education. Local evidences are limited on the assessment of diabetic patient perception on diabetic disease and self-care practice in Dilla university referral hospital, South Ethiopia, Factors influence self-care practices and Studies conducted elsewhere could not be used to infer about diabetic patients in the study area, as these differences in cultures and life style.

Therefore, the finding of the current study will be helpful to design self-care practice and to assess their perception on disease process and also it helps every staff in diabetic clinic to improve or maintain the health information about self-care practice. Thus, this study will be designed, the assessment of diabetic patient perception on diabetic disease and self-care practice in Dilla university referral hospital, South Ethiopia.

\section{Methods and Materials}

\section{Study setting}

Cross sectional study was conducted in Dilla university referral hospital, which is located about $365 \mathrm{Kms}$ to the south of Addis Ababa, the capital city of Ethiopia, and $85 \mathrm{Kms}$ to Awassa the regional capital city of SNNPR. DURH is one of the teaching hospital in Ethiopia and currently the only referral hospital in the Gedeo Zone of south part. The hospital is rapidly expanding in terms of services and provides multi-dimensional aspects of care to clients who need health service. There are five units (internal medicine, surgery, gynecology/ obstetrics, pediatrics, and psychiatry) run by the hospital. Diabetic patients get service from Diabetic clinic and admitted service also get medical wards. The service is rendered by internists, medical residents, medical interns and nurses. The study was conducted over two month from April - June, 2013.

\section{Populations}

A patients is included in the study if he/she was 18 years and older and must on follow up for a least Diabetic patients who have at least three visit on diabetic follow up units and had an appointment during the study period, patient Patients with hearing impartment or any other serious health problem who were unable to provide appropriate information were excluded.

\section{Sample size}

Sample size is calculated by using single population proportion formula by considering the following assumptions; $50 \%$ prevalence assumption, $95 \%$ confidence level of significance alpha $0.05=1.96$, and $5 \%$ margin of error, which results in the sample size of 384 . Since the source population is less than 10,000 the sample size is adjusted with correction formula and the final sample size was became 310 .

For the qualitative study part of the study using in-depth interview; ten patients with diabetes were included. The in-depth interview was conducted separately for males and females patients. The selected patients were not included in the quantitative part of the study.

\section{Sampling methods}

Health institutions available in Gedeo zone were grouped by ownership into Government and Privates. Of these, only one government hospital of Dilla university referral hospital is render diabetic follow up clinic. The lists of respondents were prepared based on their updated registration log book. After establishing the sampling frames of respondents, we would use random sampling technique to identify the study unit to be included to the survey. The eligible attending respondents were recruited in order of their appointments. Therefore, every other patients coming to the follow up clinic was interviewed until reach 310 samples. The first diabetic patient was selected by lottery method using their card number. In cases where the patients have two follow up appointments within a month, the patient's appointment date was checked and he/she was excluded from the interview.

For the qualitative part of the study participants were selected using purposive sampling technique till reached to a point of redundancy of information. The participants were interviewed after informed by principal investigator about the purpose of the study.

\section{Measurements}

Structured questionnaire is originally developed in English and this be translated to Amharic and then back translated into English by another person to ensure validity. For the validity of the questioners, it was adapted the tool from similar study done in Malaysian and also uses questioner that was prepared by Spain diabetic association and North western university of Chicago $[8,11]$. The questionnaire is slightly modified in order to fit, the purpose of this study. Each question was checked for the relationships with variables and study objectives. Pretest was carried out in Hawassa hospital. Pretest was conducted in $5 \%$ of the sample from the same source population to check clarity and consistency of the questionnaires prior to the actual data collection. Area of pretest were determine the acceptability of the questions to be asked and the methods used, reaction and willingness of the respondents, time required, performance and adequacy of data collectors and either to modify or change ambiguous and clear ideas. Far qualitative part of the study, in-depth interview was performed to get insight knowledge, perception and self-care practice.

\section{Measurement of variables}

a) Knowledge about diabetic disease: Is measured by seven items in true-false format. Correct answer will be given "1" and " 0 " is given for incorrect and don't know. Then a total score is computed out of seven marks (with the range of 0-7) the median score and those who Score above $50 \%$ have a good knowledge where as those score below $50 \%$ low knowledge.

b) Knowledge about diabetic self-care practice: Is measured by four items in true-false format. Correct answer will be given " 1 " and " 0 " is given for incorrect and don't know. Then a total score is computed out of four marks (with the range of 0-7) the median score and those who Score above $50 \%$ have a good knowledge self-care practice where as those score below $50 \%$ low knowledge self-care practice. 
c) Perception is measured by three item (with the range of 0-3) the median score is 2 and those who Score above $50 \%$ have a good Perception where as those score below 50\% poor Perception.

d) Diabetes self-care practice was measured by using items on physical exercise, diet, medication, and blood glucose measurement. Then classified the self-care as 'good self-care practice' and 'not have good self-care practice'; respondents were labeled to have "good self-care" if they scored above 50\% of the total self-care practices in the last three days.

\section{Data collection methods}

A total of four data collectors were participated in the actual data collection; they are diploma Nurses who were not working in the Diabetic follow up clinic and three supervisors who are residents in the department of internal medicine. Trained data collectors were collect the data by using a pretested and structured questioner. The investigators were give one day training to data collectors and supervisors before data collection, the training was include briefing on the general objectives of the study, discussing the contents of the questionnaires one by one and the type of information needed to handle any questions arising during data collection time; and discussions on how to maintain confidentiality and privacy. And both the principal investigator and the supervisors regularly supervise them on daily basis; there by formats was checked for its completeness, accuracy and consistency.

\section{Data quality control}

Trained data collectors who collect the data and pretesting of the instruments were made before the actual data collection. So that corrections was made accordingly. More over the principal investigator was supervise the data collection on daily basis for completeness and consistence, of the filled questionnaires. In addition to this, we were thoroughly clean and carefully enter the data to computer for commencement of analysis.

\section{Data processing and analysis}

The data was cleaned, edited, entered in to computer and analyzed using SPSS for windows program version 20. Descriptive statistics of different variables was computed to see the overall distribution of the study variable. Binary logistic regression was used for bivariate analysis and finally multiple logistic regressions were used to identify factors associated with self-care practice. To avoid many variables and unstable estimates in the subsequent model, only variables that reached a p-value less than 0.05 at bivariate analysis was kept in the subsequent model analysis. 95\% confidence interval (CI) for OR are used in judging the significance of the associations. The recorded interview were transcribed first to the language of the interview and fully translated into English. Responses and comments were grouped according to the theme and finally triangulation was performed by relating thematic areas and explaining with related to the study questions.

\section{Ethical consideration}

Dilla University, research and publication of college of Health and Medical Sciences approved this study. An official letter was written to the DURH then to Diabetic follow up clinic by DURH. Verbal consent was obtained from each respondent and the research and publication committee approved the procedure since the study was a survey and with no any harm to the respondents.

\section{Results}

\section{Socio-demographic characteristics of the study participants}

A total of 310 respondents were included in the study and giving $100 \%$ response rate. From the total participate, 200 (64.5\%) were males and $110(35.5 \%)$ were females. The mean ages of the respondent was $41.9(\mathrm{SD} \pm 1.4)$ with a range age of 18 to70 (Table1).

\begin{tabular}{|c|c|c|c|}
\hline S.No & Variable & Frequency & Percentage \\
\hline \multirow{4}{*}{1} & \multicolumn{3}{|l|}{ Sex of respondents } \\
\hline & 1.Male & 200 & 64.5 \\
\hline & 2.Female & 110 & 35.5 \\
\hline & Total & 310 & 100 \\
\hline \multirow{6}{*}{2} & \multicolumn{3}{|l|}{ Age of respondents } \\
\hline & 1. $<39$ & 112 & 36.1 \\
\hline & $2.40-44$ & 43 & 13.9 \\
\hline & $3.45-49$ & 39 & 12.6 \\
\hline & 4. $>50$ & 116 & 37.4 \\
\hline & Total & 310 & 100 \\
\hline \multirow{7}{*}{3} & \multicolumn{3}{|l|}{ Religion of respondents } \\
\hline & 1.Orthdox & 115 & 37.1 \\
\hline & 2.Catholic & 5 & 1.6 \\
\hline & 3.Protestant & 159 & 51.3 \\
\hline & 4.Muslim & 24 & 7.7 \\
\hline & 5.Other (Tradional) & $\begin{array}{c}7 \text { (3 Joba and } 4 \\
\text { traditional) }\end{array}$ & 2.3 \\
\hline & Total & 310 & 100 \\
\hline \multirow{9}{*}{4} & \multicolumn{3}{|l|}{ Ethnicity of respondents } \\
\hline & 1.Gedeo & 123 & 39.7 \\
\hline & 2.Sidama & 6 & 1.9 \\
\hline & 3.Gurage & 22 & 7.1 \\
\hline & 4.Walyeta & 11 & 3.5 \\
\hline & 5.Amhara & 81 & 26.1 \\
\hline & 6.Oromo & 51 & 16.5 \\
\hline & 7.Other & $\begin{array}{c}16 \text { (3 Gamo, } 5 \\
\text { hadiya and } 8 \text { cafa) }\end{array}$ & 5.2 \\
\hline & Total & 310 & 100 \\
\hline \multirow{9}{*}{5.} & \multicolumn{3}{|c|}{ Marital status of respondents } \\
\hline & 1.Single & 77 & 24.8 \\
\hline & 2.Married \& live together & 206 & 66.5 \\
\hline & 3.Married but not live & 12 & 3.9 \\
\hline & Together & & \\
\hline & 4.Divorced & 0 & \\
\hline & 5.Widowed & 15 & 4.8 \\
\hline & 6.Other & & \\
\hline & Total & 310 & 100 \\
\hline \multirow{8}{*}{6} & \multicolumn{3}{|l|}{ Living with } \\
\hline & 1. My Spouse & 213 & 68.7 \\
\hline & 2.Alone & 50 & 16.1 \\
\hline & 3.Family & 19 & 6.1 \\
\hline & 4.Father & 8 & 2.6 \\
\hline & 5.Mother & 5 & 1.6 \\
\hline & 6.Childeren & 15 & 4.8 \\
\hline & Total & 310 & 100 \\
\hline \multirow{7}{*}{7} & \multicolumn{3}{|l|}{ Educational status } \\
\hline & 1.Illiterate & 39 & 12.6 \\
\hline & 2.Grade 1-8 & 76 & 24.5 \\
\hline & 3. Grade $9-10$ & 28 & 9 \\
\hline & 4. preparatory & 41 & 13.2 \\
\hline & 5.Higher Education & 126 & 40.6 \\
\hline & Total & 310 & 100 \\
\hline
\end{tabular}




\begin{tabular}{|c|c|c|c|}
\hline & Occupation status & & \\
\hline & 1.goverment Employ & 117 & 37.7 \\
\hline & 2.Private Employ & 7 & 2.3 \\
\hline & 3.Merchant & 29 & 9.4 \\
\hline & 4.Farmer & 53 & 17.1 \\
\hline 8 & 5.Driver & & \\
\hline & 6.Daily laborer & 8 & 2.6 \\
\hline & 7.Unemployed & 26 & 8.4 \\
\hline & 8.Other & $\begin{array}{l}70 \text { ( } 8 \text { house wife, } 13 \\
\text { pension, } 49 \text { student) }\end{array}$ & 22.6 \\
\hline & Total & 310 & 100 \\
\hline 9 & Residence of subjec & & \\
\hline & 1.Urban & 93 & 30 \\
\hline & 2.Rural & 217 & 70 \\
\hline & Income level & & \\
\hline & 1.low level & 125 & 40.3 \\
\hline 10 & 2.medium level & 101 & 32.6 \\
\hline & 3. High level & 84 & 27.1 \\
\hline & Total & 310 & 100 \\
\hline
\end{tabular}

Table 1: Socio-demographic characteristics of patients with diabetes in Dilla referral hospital, 2014

\begin{tabular}{|c|c|c|c|}
\hline S.No & Variable & Frequency & Percentage \\
\hline \multirow{4}{*}{1.} & \multicolumn{3}{|l|}{ Type of DM } \\
\hline & 1.Type I & 123 & 39.7 \\
\hline & 2.Type II & 187 & 60.3 \\
\hline & Total & 310 & $100 \%$ \\
\hline \multirow{6}{*}{2.} & \multicolumn{3}{|l|}{ Duration of DM } \\
\hline & 1. $<1 \mathrm{Yr}$ & 84 & 27.1 \\
\hline & 2.1-2 Yr & 47 & 15.2 \\
\hline & 3.3-5 Yr & 74 & 23.9 \\
\hline & 4. $>5 \mathrm{Yr}$ & 105 & 33.9 \\
\hline & Total & 310 & 100 \\
\hline \multirow{4}{*}{3.} & \multicolumn{3}{|l|}{ Follow Up of DM } \\
\hline & 1.Always & 115 & 37.1 \\
\hline & 2.Sometimes & 195 & 62.9 \\
\hline & Total & 310 & 100 \\
\hline \multirow[t]{4}{*}{9.} & \multicolumn{3}{|c|}{ Average of three consecutive blood glucose level } \\
\hline & 1.Normal (<FBG127) & 44 & 14.2 \\
\hline & 2.Abnormal (>FBG127) & 266 & 85.8 \\
\hline & 3. Total & 310 & 100 \\
\hline
\end{tabular}

Table 2: Clinical Characteristics and related history of patients with diabetes in Dilla university referral hospital, 2014

\section{DM related history of study participants}

Among the total respondents, 187 (60.3\%) of them were medically diagnosed with type- 2 diabetes. The mean duration since medically diagnosed for diabetes was $2.5(\mathrm{SD} \pm 1.40)$ years. The majority 195 (62.9\%) of the study participant did not have follow up for their health impairment. As result a substantial proportion 266 (85.5\%) patients had abnormal blood glucose level or glycemic control (Table 2).

\section{Perception of patients towards diabetes mellitus}

Among the total participants, the majority 242 (78.1\%) of patients had positive Perception towards DM and diabetes complications. More than half of the respondent 174 (56.1\%) perceives or agree that DM never get break and the remaining 105 (35.2\%) were not agree. Regarding severity of diabetes and its related complications, a total of
$215(65.4 \%)$ respondents were agreed on DM is very serious disease. about half, $162(52.3 \%)$ participants were disagreed on DM changes personal outlook in life and the remaining 128 (41.3\%) were agreed (Table 3).

\section{Patient diabetic self-care practice among study participants}

We measured diabetes self-care practice using the following recommended self-care items like physical exercise, diet, medication and blood glucose measurement. We classified the self-care as 'good self-care practice' and 'not good self-care practice'; respondents were labeled to have "good self-care" if they scored above 50\% of the total self-care practices.

Among the total respondents, majority 238 (76.8\%) of them had good practiced on the recommended self-care practices. Almost half of the respondents 154 (49.7\%) followed the recommended dietary intake for controlling DM. Only 138 (44.5\%) had exercise for average of 30 minutes per day. Regarding blood glucose monitoring only $62(20 \%)$ of patients measured their blood glucose by themselves one per day. Almost all of the respondents 289 (93.2\%) had taken the prescribed drugs appropriately whereas $21(6.8 \%)$ did not take the prescribed drugs as prescribed (Table 4).

\section{Self-care practice on blood glucose monitoring and it's predictors among study participants}

Among the total respondents, $62(20 \%)$ of patients reported that they performed self-measuring for blood glucose. Almost 35 (55.5\%) of the patients did not control their blood glucose regularly ,According to the result of the multivariate analysis, individuals' with high income levels were 5.8 times more likely to perform self-blood glucose monitoring than less income levels [OR-5.83, 95\% CI $(2.41,14.12)]$. On the other side, diabetic patients with medium income level were 2.99 times more likely to perform self-blood glucose monitoring than less income level [OR-2.99, 95\% CI $(1.32,6.78)]$. Diabetic patients who were type one were 4.2 times more likely performed self-blood glucose monitoring than type two patients (AOR: $4.17,95 \%$ CI: 2.06-8.45).

\begin{tabular}{|c|c|c|c|}
\hline S.No & Variable & Frequency & Percent \\
\hline 1 & $\begin{array}{l}\text { General Perception score towards DM } \\
\text { 1. positive perception } \\
\text { 2. negative perception } \\
\text { Total }\end{array}$ & $\begin{array}{c}242 \\
68 \\
310\end{array}$ & $\begin{array}{l}78.1 \\
21.9 \\
100\end{array}$ \\
\hline 2 & $\begin{array}{l}\text { You never get a break from DM } \\
\text { 1.Agree } \\
\text { 2.Disagree } \\
\text { 3.Neutral } \\
\text { Total }\end{array}$ & $\begin{array}{c}174 \\
109 \\
27 \\
310\end{array}$ & $\begin{array}{c}56.1 \\
35.2 \\
8.2 \\
100\end{array}$ \\
\hline 3 & $\begin{array}{l}\text { DM changes personal outlook in life } \\
\text { 1.Agree } \\
\text { 2.Disagree } \\
\text { 3.Neutral } \\
\text { Total }\end{array}$ & $\begin{array}{l}128 \\
162 \\
20 \\
310\end{array}$ & $\begin{array}{l}41.3 \\
52.3 \\
6.5 \\
100\end{array}$ \\
\hline 4 & $\begin{array}{l}\text { DM is very serious disease } \\
\text { 1.Agree } \\
\text { 2.Disagree } \\
\text { 3.Neutral } \\
\text { Total }\end{array}$ & $\begin{array}{l}215 \\
74 \\
21 \\
310\end{array}$ & $\begin{array}{c}69.4 \\
23.9 \\
6.8 \\
100\end{array}$ \\
\hline 5 & $\begin{array}{l}\text { Perceived Health Status of the respondent } \\
\text { 1.Exellent } \\
\text { 2.V.good } \\
\text { 3.good } \\
\text { 4. Medium } \\
\text { 5.Dangerous } \\
\text { Total }\end{array}$ & $\begin{array}{c}11 \\
53 \\
178 \\
57 \\
11 \\
310\end{array}$ & $\begin{array}{c}3.5 \\
17.1 \\
57.4 \\
18.4 \\
3.5 \\
100\end{array}$ \\
\hline
\end{tabular}

Table 3: Perception of Patients with diabetes on Self-care practice in Dilla university referral hospital, 2014 


\begin{tabular}{|l|l|c|}
\hline S. No & \multicolumn{1}{|c|}{ Variable } & N (\%) \\
\hline 1 & $\begin{array}{l}\text { DM self- care practice scores levels: }(\mathrm{n}=310) \\
1 . \quad \text { Having self-care practice } \\
2 . \quad \text { Not have self- care practice }\end{array}$ & $\begin{array}{c}238(76.8 \%) \\
72(23.2 \%)\end{array}$ \\
\hline 2 & $\begin{array}{l}\text { Patients self-blood glucose monitoring practice: }(\mathrm{n}=310) \\
\text { 1.Yes 2.No }\end{array}$ & $\begin{array}{c}62(20 \%) \\
248(80 \%)\end{array}$ \\
\hline 3 & $\begin{array}{l}\text { Patients Self-care practice of insulin intake to control DM: } \\
(\mathrm{n}=310) \text { 1.Yes2.No }\end{array}$ & $\begin{array}{c}90(29 \%) \\
220(71 \%)\end{array}$ \\
\hline 4 & $\begin{array}{l}\text { Patients' physical exercise to control DM: }(\mathrm{n}=310) \\
\text { 1.Yes 2.No }\end{array}$ & $\begin{array}{c}138(44.5) \\
172(55.5)\end{array}$ \\
\hline 5 & $\begin{array}{l}\text { Patients' Regular Dietary Adjustment to control DM: } \\
(\mathrm{n}=310)\end{array}$ & $154(49.7 \%)$ \\
1. Yes 2. No & $156(50.5 \%)$ \\
\hline 6 & $\begin{array}{l}\text { Patients Self-care practice on taking medication } \\
\text { adherence } \\
\text { 1. Taken as prescribed by physician } \\
\text { 2. not follow as prescribed by physician }\end{array}$ & $289(93.2)$ \\
\hline
\end{tabular}

Table 4: Patient self-care practice among patients with diabetes in Dilla university referral hospital, 2014.

\begin{tabular}{|c|c|c|c|c|}
\hline \multirow[t]{2}{*}{ S.No } & \multirow[t]{2}{*}{ Variable } & \multicolumn{2}{|c|}{$\begin{array}{c}\text { Patients self-blood } \\
\text { glucose monitoring } \\
\text { practice: } \mathbf{N}(\%)\end{array}$} & \multirow[t]{2}{*}{ COR (Cl) } \\
\hline & & yes & No & \\
\hline 1 & $\begin{array}{l}\text { Income level of the respondents } \\
\text { 1. Low level } \\
\text { 2. Medium level } \\
\text { 3. Highest level }\end{array}$ & $\begin{array}{l}14(4.5) \\
26(8.4) \\
22(7.1)\end{array}$ & $\begin{array}{l}111(35.8) \\
75(24.2) \\
62(20.2)\end{array}$ & $\begin{array}{c}1 \\
2.99(1.32-6.78) \\
5.83(2.41-14.12)\end{array}$ \\
\hline 2 & $\begin{array}{l}\text { Type of DM } \\
\text { 1. Type } 1 \\
\text { 2. Type } 2\end{array}$ & \begin{tabular}{|c|}
$39(12.6)$ \\
$23(7.1)$
\end{tabular} & $\begin{array}{c}84(27.1) \\
164(52.9)\end{array}$ & $\begin{array}{c}4.17(2.06-8.45) \\
1\end{array}$ \\
\hline 3 & $\begin{array}{l}\text { Perception of DM } \\
\text { 1. Correct perception } \\
\text { 2. Incorrectperception }\end{array}$ & $\begin{array}{l}59(19) \\
3(1.0)\end{array}$ & $\begin{array}{c}183(59) \\
65(21)\end{array}$ & $\begin{array}{c}4.86(1.40-16.90) \\
1\end{array}$ \\
\hline 4 & $\begin{array}{l}\text { General knowledge of DM } \\
\text { 1. Low knowledge of DM } \\
\text { 2. Good knowledge of DM }\end{array}$ & $\begin{array}{c}6(1.9) \\
56(18.1)\end{array}$ & $\begin{array}{c}58(18.7) \\
190(61.3)\end{array}$ & $\begin{array}{c}1 \\
1.33(0.31-5.74)\end{array}$ \\
\hline 5 & $\begin{array}{l}\text { Knowledge about diabetes self-care } \\
\text { 1. Low Knowledge about diabetes self-care } \\
\text { 2. Good Knowledge about diabetes self-care }\end{array}$ & $\begin{array}{c}7(2.4) \\
53(17.8)\end{array}$ & $\begin{array}{l}84(28.3) \\
153(51.5)\end{array}$ & $\begin{array}{c}1 \\
2.74(0.86-8.72)\end{array}$ \\
\hline 6 & $\begin{array}{l}\text { Information received about DM } \\
\text { 1. Yes } \\
\text { 2. No }\end{array}$ & $\begin{array}{c}24(7.7) \\
38(12.3)\end{array}$ & \begin{tabular}{|l|}
$59(19.0)$ \\
$189(61)$
\end{tabular} & $\begin{array}{c}2.27(1.13-4.58) \\
1\end{array}$ \\
\hline
\end{tabular}

Table 5: Multivariate analysis for Predictors of Self-care Practice on Blood Glucose Monitoring among patients with diabetes in Dilla university referral hospital, 2014.

Individuals who had positive perception towards diabetes disease were 4.9 times more likely to perform self-blood glucose monitoring than negative perception [OR-4.86, 95\% CI $(1.40,16.90)]$. In addition, patients with frequently received information on DM were two times more likely to perform self-blood glucose monitoring [OR- $2.27,95 \%$ CI $(1.13,4.58)]$ than patients who did not received information about the disease. However, in multivariate analysis knowledgeable on DM and knowledgeable on self-care did not show significant relation with self-blood glucose monitoring practice (Table 5).

\section{Self-care practice on physical exercise and it's predictors among study participants}

Among the total respondents, $138(44.5 \%)$ patients had different type of physical exercise to control DM. Of which $122(79.2 \%)$ of patients exercised walking and followed by 27 (17\%) gymnastic. Among those who had walking, 90 (73.8\%) of patients had been walking on foot with in an average of forty minutes interval.

In multivariate analysis, male diabetic patients were two times more likely to have physical exercise than females, (AOR: 2.32, 95\% CI: 1.32-4.06). Individuals who had less than one and 3-5 years duration of DM were around 2.26and 2.4 times more likely to perform exercise than those who had greater than five years duration of DM [OR2.26, 95\% CI (1.14-4.49)] and [OR2.40, 95\% CI (1.18-4.87)] respectively. On the other hand, diabetic patients who were good knowledgeable on DM were two times more likely to perform exercise than with low knowledgeable on DM [OR 2.12, 95\% CI $(1.02,4.38)]$. Lastly occupational statuses of respondents showed significant relation with performing exercise (Table 6).

\section{Patients' dietary adjustment for recommended foods intake and its predictors}

regarding regular dietary adjustment of recommended food intake, almost half 154 (49.7\%) of them had regular dietary adjustment intake. The recommended dietary adjustment intake time for patients with diabetes were (breakfast, lunch, snack and dinner) and different types of food which was recommended for patients with diabetes (vegetables, cereals, rice, wheat and its products, potatoes and sweet potatoes, and fruits). Among those who had regular dietary intake time, 139 (27.2\%) of them had regular time adjustment for breakfast followed by dinner $131(25.6 \%)$. Less proportion $51(16.5 \%)$ of them answered that taken special diet when blood glucose level was high such as Besso 18 followed by Shiferraw.

In multivariate analysis, male diabetic patients were two times more likely to have diet adjustment than females, (AOR: 2.17, 95\% CI: 1.25, 3.76). On the other hand, diabetic patients with very high income were 2.5 times more likely to have diet adjustment than with less income [OR-2.49, 95\% CI $(1.25,4.94)]$. In addition, patients with frequent received information on DM were nearly three times more likely to have diet adjustment [OR- 2.94, 95\% CI $(1.59,5.42)]$ than patients with no received information about DM disease. Individuals who had 3-5 years of duration of DM were 0.5 times less likely to have diet adjustment than those who had less than one year's duration of DM [OR 0.47, 95\% CI (0.23 -0.95)]. Age of respondents showed significant relation with diet adjustment (Table 7).

\section{Patients' received health information about DM}

Less than half $83(26.8 \%)$ of the surveyed patients had been received information about DM since their diagnosis. Surveyed patients cited that doctors and nurses are the primary source of information for $\mathrm{DM}$, but majority of surveyed patients mentioned that there was no

\begin{tabular}{|c|c|c|c|}
\hline S.No & Variable & Frequency & Percentage \\
\hline \multirow{4}{*}{1} & Having Physical exercise & & \\
\hline & 1.Yes & 138 & 44.5 \\
\hline & 2.No & 172 & 55.5 \\
\hline & Total & 310 & 100 \\
\hline \multirow{5}{*}{2} & Type of physical Exercise & & \\
\hline & 1.Waking & 122 & 79.2 \\
\hline & 2.Swming & 5 & 3.2 \\
\hline & 4.Gymnastic & 27 & 17.5 \\
\hline & 5.Total & 154 & 100 \\
\hline \multirow{5}{*}{3} & How long do u wake & & \\
\hline & 1. $<40 \mathrm{~min}$ & 90 & 73.8 \\
\hline & $2.40-60 \mathrm{~min}$ & 26 & 21.3 \\
\hline & 3.> $60 \mathrm{~min}$ & 6 & 4.9 \\
\hline & Total & 122 & 100 \\
\hline
\end{tabular}

Table 6: Patients' physical exercise to control DM among patients with diabetes in Dilla university referral hospital, 2014. 


\begin{tabular}{|c|c|c|c|}
\hline S.No & Variable & Frequency & Percentage \\
\hline \multirow{4}{*}{1} & Do You have diet adjustment & & \\
\hline & 1.Yes & 154 & 49.7 \\
\hline & 2.No & 156 & 50.3 \\
\hline & Total & 310 & 100 \\
\hline \multirow{6}{*}{2} & Which meals & & \\
\hline & 1.Breakfast & 139 & 27.2 \\
\hline & 2.Lunch & 125 & 24.5 \\
\hline & 3.Snak & 116 & 22.7 \\
\hline & 4.Dinner & 131 & 25.6 \\
\hline & Total & 511 & 100 \\
\hline \multirow{4}{*}{3} & Special diet when Blood Glucose level is high & & \\
\hline & 1.Yes & 51 & 16.5 \\
\hline & 2.No & 259 & 83.5 \\
\hline & Total & 310 & 100 \\
\hline \multirow{5}{*}{4} & Which type of diet? & & \\
\hline & 1.Beso & 18 & 35.3 \\
\hline & 2.kollo & 8 & 15.7 \\
\hline & 3.shiferaw & 10 & 19.6 \\
\hline & $\begin{array}{l}\text { 4.Sugar } \\
\text { Total }\end{array}$ & $\begin{array}{l}15 \\
51\end{array}$ & $\begin{array}{l}29.4 \\
100\end{array}$ \\
\hline
\end{tabular}

Table 7: Patients' Dietary Adjustment for recommended foods to control DM among patients with diabetes in Dilla university referral hospital, 2014

\begin{tabular}{|c|c|c|c|}
\hline S.No & Variable & Frequency & $\%$ \\
\hline \multirow{5}{*}{1} & Do You receive information about DM & & \\
\hline & 1.Yes & 83 & 26.8 \\
\hline & 2.No & 224 & 72.3 \\
\hline & 3.Not sure & 3 & 1 \\
\hline & Total & 310 & 100 \\
\hline \multirow{5}{*}{2} & If yes, what is the Topic & & \\
\hline & 1.About Foot Care & 58 & 28.7 \\
\hline & 2.About diet & 78 & 38.6 \\
\hline & 3.About Physical exercise & 66 & 32.7 \\
\hline & 4.Total & 202 & 100 \\
\hline \multirow{5}{*}{3} & Reason for not received information on DM & & \\
\hline & 1. No HE about DM & 161 & 64.1 \\
\hline & 2. Busy schedule of health care provider & 57 & 22.7 \\
\hline & $\begin{array}{l}\text { 1. un readiness of health care provider to provide } \\
\text { information }\end{array}$ & 33 & 13.4 \\
\hline & Total & 251 & 100 \\
\hline \multirow{6}{*}{4} & Which Topic is more clear for You & & \\
\hline & 1.About foot care & 54 & 22 \\
\hline & 2.About diet & 72 & 29.4 \\
\hline & 3.About Exercise & 58 & 23.7 \\
\hline & 4.About drug intake & 61 & 24.9 \\
\hline & Total & 245 & 100 \\
\hline
\end{tabular}

Table 8: Patients' received Health information about DM among patients with diabetes in Dilla university referral hospital, 2014.

formal health education on DM. The available scientific knowledge concerning diabetes mellitus is an important resource to guide and educate diabetes patients concerning self-care. Self-care concepts that can benefit patients include adherence to diet, physical activity, blood glucose monitoring, and taking oral medication and insulin. For those among Surveyed patients, 78 (38.8\%) had been received information about diet followed by exercise $66(32.7 \%)$ during their diagnosis. Among those who had not received information concerning self-care and information about DM, the most commonly mentioned reasons were unavailability of health education which accounted $161(64.1 \%)$ followed by busy Schedule of health care provider which accounted $57(22.7 \%)$ (Table 8$)$.

\section{Discussion}

This study has tried to assess diabetic patient perception on diabetic disease and self-care practice in Dilla university referral hospital, South Ethiopia. This study revealed that $79.4 \%$ of them were knowledgeable about diabetes this finding is consistent with a study done in Bangladesh (75\%) (38). whereas the finding from Harer is incomparable to our study where $(93.7 \%)$ is knowledgeable about DM this might be because of health education/information given in our study area is only 59 (19\%) (39). Contrary to this Scenario study from Egypt finding show that $(90 \%)$ were poor knowledgeable about diabetes. With regard to knowledge on DM symptoms and diabetes self-care practices, $52.1 \%$ and $69.1 \%$ of them were knowledgeable on DM symptoms and diabetes self-care practices respectively but this finding was incongruent with a study done in Harer (88.3\% and $93.2 \%)$ regarding knowledgeable on DM symptoms and diabetes self-care practices respectively. The cause for the discrepancy may be the same with knowledge gap created between Harer and our study area with similar pattern self-care result is lower in our study area than the studies done in Sewden 56\%.

This means that health care setting, communities, service providers and patients with diabetes should work together to manage the disease. This finding is consistent to qualitative finds according to one in-depth interview respondents said that "We need to be work holistically and work hand in hand with one another as a health team". This approach helps to explores patients' understanding of the disease, treatment options and self-care practice. Successful chronic disease management is dependent on effective, systematic and interactive communication between patients and service providers as well as the health system with which they make contact.

In this study patient showed understanding of the causes and complications of the disease and most of (78.1\%) them developed positive perception towards DM. However this finding differs where a study conducted in United Arab Emirates (72\%) had a negative attitude towards having diabetes [12]. Finding from United Arab Emirates is supported by our qualitative study according to respondent from indepth result one female participant said that "DM patient always ask themselves the following questions after the diagnosis: Why me? What am I going to eat? What are the people going to say about me? Where will I get money to buy food?" This question that comes to the mind of patient may make a patent to perceive negatively. It is therefore that patients should be encouraged to understand the meaning of chronic disease and its management. This means that Patients with positive beliefs will have positive attitudes towards behavioral change and will be motivated to comply, and those with negative will be less motivated and will resist behavior change. In this study individuals who were positive perception towards diabetes disease were 2.7 times more likely to perform recommended self-care than negative perception (OR-2.74, $95 \%$ CI $(1.27,5.91)$. Similarly a study conducted in Jimma university hospital Patients with high perceived severity of the disease was more likely to adhere to self-care practice [13].

The available scientific knowledge and perception concerning diabetes mellitus is an important resource to guide and educate diabetes patients concerning self-care. Self-care concepts that can benefit patients include adherence to diet, physical activity, blood glucose monitoring and taking oral medication and insulin [6,7]. In the current study, 238 (76.8\%) of them had good practiced on the recommended self-care practices. Among the recommended self-care behaviors, drug adherence 289 (93.2\%), dietary intake 154 (49.7\%) and regular exercise 138 (44.5\%) were the most practiced recommended self-care. On the other hand, self-blood glucose monitoring was the least practiced 
which accounted $62(20 \%)$. In this study, the recommended self-care practice was higher $(76.8 \%)$ where compare to a study conducted in Harer (39.2\%) and in United Arab Emirates (37.7\%). With regarded to specific recommended self-care practices, dietary intake $154(49.7 \%)$ and regular exercise $138(44.5 \%)$ were less likely practiced with compare to a study conducted in Harer [12,14]. In this study there was a consistence finding from in-depth interview according to one female DM patient she said that "some patients knew about the recommended food practices, but because of socio-economic barriers (lack of finances) were unable to acquire the right kind of food. Some of the challenges to dietary adherence involve avoiding favorite foods, selecting healthful alternatives, time management (patients find it difficult to plan food with insulin or oral medication) and social support (as most women prepare food for their families).

For successful diabetes management individuals should pay more attention for knowledge on the recommended self-care practices. The gain of knowledge on management of diabetes is to help patients to make life skill changes and offer the support needed to achieve optimal health [15]. In line with in the current study patients who were knowledgeable on DM self-care 6.52 were times more likely performed recommended self-care (OR-6.52, 95\% CI $(2.88,14.78)$ than patients who were low knowledgeable on DM self-care. This finding was supported the studies conducted in Bangladesh and United Arab Emirates knowledge was significant independent predictors of good practice (OR- 1.28 (95\% CI: 1.03 to 1.60$)$ and $(r=0.320, p, 0.001)$ respectively $(38,55)$. However, in this study diabetic patients who were knowledgeable on DM were 0.3 times less likely performed recommended self-care to manage the disease (OR-0.29, 95\% CI $(0.10,0.80)$.

On the other hand, individuals with educational level of grade 9-12 and higher educational status were more likely to adhere on selfcare practice than patients who were illiterate (OR-3.96, 95\% CI $(1.25$, $12.55))$ and [OR-3.45, 95\% CI $(1.16,10.29)]$ respectively. Similar results were found in other studies in Harer, and Egypt $[14,16]$ educational statuses were more likely to adhere on self-care practice.

However in the current study patients with less frequent information received on DM did not show significant relation with selfcare practice to control DM, but a study conducted in Harer, patients with less frequent information received were less likely performed selfcare (OR- $0.3,95 \%$ CI $(0.09,0.79)$. This finding was strongly support indepth interview he said that "There was the problem of limited time for consultation with the health profession was seen as one of the possible problems that could contribute to patients being non-adherent in their management. Another participants expressed the view that 'we need a patient-centered approach health care providers can give care that is more effective over time".

In this study, several explanations were possible for the fact that respondents had knowledge of DM but inappropriate self-care practices. The results of this study encourage a positive outlook for requiring a diabetes education during every visit and counseling that have an impact in improving the perception about disease, knowledge on DM, Self-care practice that include adherence to diet, physical activity, blood glucose monitoring and taking medication. This is supported with in-depth findings; "one participant said that Patients need to be encouraged always to 'think out of the box' in order to discourage them from negative thoughts. 'Patients need to cut down their food portions, they also need to exercise; but they need to know what is regarded as sufficient exercise or physical activity and how much is sufficient".

\section{Limitations}

The finding of this study may be interpreted with caution as it is facility -based which may produce more. Self-reported about self-care practice, self-reported dietary habits.

\section{Conclusion}

In this study (78.1\%) diabetic patients were developed positive perception towards DM and has a significant effect for patients with diabetes to provide own self-care practice. Two third, 238 (76.8\%) of them had good practiced on the recommended self-care practices. Among the recommended self-care behaviors, drug adherence 289 (93.2\%), dietary intake 154 (49.7\%) and regular exercise 138 (44.5\%) were the most practiced recommended self-care. Self-blood glucose monitoring was the least practiced which accounted 62 (20\%). Majority $79.4 \%$ of the respondents were knowledgeable about diabetes, but those diabetic patients who were knowledgeable on DM were less likely performed recommended self-care to manage the disease. On other hand those diabetic patients who were knowledgeable on DM selfcare were more likely performed recommended self-care. Education also has a significant effect for patients with diabetes in order to provide own self-care practice. From this study we can also conclude that income level, perception towards DM and patients who received information were more likely to adhere to self-care practice of SMBG. This finding also showed that duration of DM and knowledgeable about diabetes has significant statistical effect on self-care behavior of physical exercise. Patient's income also another determining factor for diabetes self-care practices of diet adjustment. Patients with regularly received information and duration of DM were more likely to adhere to self-care practice of diet adjustment. Lastly A major point to address therefore is regular access to/contact with diabetic educators which currently is severely substandard.

\section{References}

1. Lawal M (2008) Management of diabetes mellitus in clinical practice. Br J Nurs 17: 1106-1113.

2. American Diabetic Association (2008) Standard of medical care in diabetes Diabetes care 31:43.

3. Mensing C, Boucher J, Cypress M, Weinger K, Mulcahy K, et al. (2005) National standards for diabetes self-management education. Diabetes Care 28 Suppl 1: S72-79

4. Lin C, Anderson R, Hagerty B, Lee B (2007) Diabetes self management experience among Taiwanese patients with type two diabetes mellitus. J Clin Nurs 9: 1-3.

5. Tan MY, Magarey J (2008) Self-care practices of Malaysian adults with diabetes and sub-optimal glycaemic control. Patient Educ Couns 72: 252-267.

6. WHO (2003) Diabetes estimates and projection. Geneva

7. WHO (2005) World Health Organization Health Action in Crises, Ethiopia Strategy Paper.

8. Lester FT (1991) Clinical status of Ethiopian diabetic patients after 20 years of diabetes. Diabet Med 8: 272-276.

9. Feleke $Y$, Enquselassie $F(2005)$ An assessment of the health care system for diabetes in Addis Ababa. Ethiop J Health dev 19: 203-210.

10. Peyrot M, Rubin RR, Lauritzen T, Snoek FJ, Matthews DR, et al. (2005) Psychosocial problems and barriers to improved diabetes management: results of the Cross-National Diabetes Attitudes, Wishes and Needs (DAWN) Study. Diabet Med 22: 1379-1385.

11. Harris MI, Klein R, Welborn TA, Knuiman MW (1992) Onset of NIDDM occurs at least 4-7 yr before clinical diagnosis. Diabetes Care 15: 815-819.

12. Mshunqane N, Stewart AV, Rothberg AD (2012) Type 2 diabetes management: Patient knowledge and health care team perceptions, South Africa. Afr J Prm Health Care Fam Med. 4: 7

13. Lawal M (2008) Management of diabetes mellitus in clinical practice. Br J Nurs 17: $1106-1113$

14. Gillibrand R, Stevenson J (2006) The extended health belief model applied to the experience of diabetes in young people. Br J Health Psychol 11: 155-169. 
Citation: Addisu Y, Eshete A, Hailu E (2014) Assessment of Diabetic Patient Perception on Diabetic Disease and Self-Care Practice in Dilla University Referral Hospital, South Ethiopia. J Metabolic Synd 3: 166. doi:10.4172/2167-0943.1000166

15. Tan MY, Magarey J (2008) Self-care practices of Malaysian adults with diabetes and sub-optimal glycaemic control. Patient Educ Couns 72: 252267.
6. Mohammed A, Haddad M (2008) Economic, Social and Clinical evaluations of diabetes self-management program at Universiti Sains Malaysia Health Center: Comparison between structured and less structured educational programs. 